\title{
European Breast Cancer Conference 12, October 2-3, 2020: Interview with the Chairs
}

\author{
Rachel Wuerstlein $^{a}$ Nadia Harbeck ${ }^{a}$ Tanja Spanic ${ }^{b, c}$ Emiel J.T. Rutgers ${ }^{d}$ \\ Javier Cortes ${ }^{e}$ \\ a Breast Center, Department of Obstetrics and Gynecology, and Comprehensive Cancer Center (CCC Munich LMU), \\ University Hospital, LMU Munich, Munich, Germany; ${ }^{b}$ Europa Donna - the European Breast Cancer Coalition, \\ Milan, Italy; ${ }^{\mathrm{C} E u r o p a ~ D o n n a ~ S l o v e n i a, ~ L j u b l j a n a, ~ S l o v e n i a ; ~}{ }^{\mathrm{d}}$ Department of Surgery, Netherlands Cancer Institute, \\ Amsterdam, The Netherlands; ${ }^{\mathrm{E}} \mathrm{IOB}$ Institute of Breast Cancer, Quiron Group, Barcelona, Spain
}

With a short interview to the very recent European Breast Cancer Conference 12 (EBCC12), the first-ever virtual EBCC 2020, we want to honor the input of this conference to our daily clinics and translational research in Europe. With more than 2,500 participants and over 6,000 logins over the two days and more than 450 presentations of over 130 speakers, this conference represented a large overview and, with the latest abstracts, an actual insight into the interdisciplinary and interprofessional setup of the EBCC, organized by ESMO, EUSOMA, and Europa Donna. Login on the virtual platform with access to all the presentations and discussions will be available for all participants until end of this year. All information can be found at www.eortc.org.

We here present feedback from the chairs of this conference: Nadia Harbeck (Germany), Tanja Spanic (on behalf of Europa Donna), Emiel J.T. Rutgers (The Netherlands), and the local chair Javier Cortes (Spain).

\section{Wuerstlein: What makes the EBCC 2020 unique?}

Harbeck, Rutgers, Cortes: The EBCC is a unique breast cancer conference as it addresses physicians and nurses from all different specialties together with patients. 2020 was the first EBCC ever held in a completely virtual for- mat - we had originally planned the conference as a live event in Barcelona in March of 2020 and then had to change into October 2-3, first intended still as a live meeting and then changing into virtual due to the still ongoing COVID-19 pandemic. Despite the logistical changes, we had 5 late breaking abstracts and 454 recorded and live presentations presenting best and newest breast cancer research. So in my opinion this demonstrates very clearly that despite these difficult pandemic times, the motivation to advance the field in the interest of our patients and to connect in a multidisciplinary interprofessional way is greater than ever. As chairs of the conference, we thank all speakers and chairs, the organizational staff, as well as the industry sponsors for their dedication and continued support throughout the change from an on-site to a virtual format.

Spanic: The EBCC 2020 is unique in that it is the first virtual EBCC that the partners EUSOMA, EORTC, and Europa Donna have held. It is also unique in that it is being held during a pandemic which has had an enormous impact on breast services throughout the world. The coverage of the response of the scientific, research, and patient community to this immediate problem is timely and extremely important and provided great added value to the themes which are usually covered in this conference, 
which has brought together breast cancer specialists, medical researchers, and patient organizations for what is now the 12th every other year edition.

As we have seen with other similar events which had to go virtual in the past months, holding a conference virtually allows many more attendees (over 2,500 from 80 different countries) and has the definite advantage of going over the sessions at will for a given time after the conference.

\section{Wuerstlein: What is/was your personal highlight of the EBCC12?}

Harbeck, Cortes: Our personal highlight was the fantastic motivation and dedication of every single speaker and chair to make this a successful congress. We had two great keynote speeches, one on patient-reported outcomes by Dr. Amylou Dueck, the other on endocrine resistance by Prof. Nicholas Turner. I would like to congratulate again Dr. Fatima Cardoso on the European Breast Cancer Science Award. She gave a very interesting talk on "Research and education in oncology: Two sides of the same coin." Last but not least, we had a very important manifesto session again "Why don't all European breast cancer patients get the most appropriate care?" This manifesto has always been a special part of the EBCC since it demonstrates that we not just have a responsibility for our own patients or our own research, but also a political responsibility to raise our voices in order to create equal access to optimal care in Europe and beyond its borders.

Spanic: One of the highlights was a very current topic - the influence of COVID-19 on breast cancer care - and definitely the opening lecture of Dr. Fatima Cardoso on the European Breast Cancer Science Award. Beside that we found the manifesto session very enlightening from many points of view, especially the honest sharing on the part of professionals engaged in breast cancer care concerning the issues and barriers that exist to providing state-of-the-art breast services despite excellent guidelines etc.

Imaging experts, pathologists, surgeons and radiation and medical oncologists along with patients all acknowledged the "elephant in the room" consisting of outdated, impractical, unrealistic reimbursement policies. The call for change does not require health care systems to invest more money, but simply to allocate it in a more cost-efficient way.

I also think that the session dedicated to explaining the European Commission Initiative on Breast Cancer (ECIBC) guidelines, the ratio behind their creation, the work that has been carried out until now, and the future developments expected with the rollout of the QA scheme is also a highlight of the Conference from the perspective of a patients' advocate organization, whose mission is to disseminate evidence-based reliable information covering what has been done and what needs to be done in $\mathrm{Eu}-$ rope and then in each European country.

\section{Wuerstlein: What is your feedback for this year's EBCC as a virtual conference?}

Harbeck, Rutgers, Cortes: We think it went great and we saw some clinically very relevant data. The chat discussions also allowed the audience to participate and ask questions - in the debates we saw how you can also interact quite vividly in a virtual format. Nevertheless, a virtual conference cannot replace the face-to-face communication of a live conference with its casual meetings in the coffee breaks and the discussions with the speakers after their sessions. In particular, for a multidisciplinary interprofessional meeting such as the EBCC these networking opportunities as well as the live exchange between patients and caregivers is crucial, so we hope that we can all meet in person again in March 2022 at the EBCC13 in Barcelona.

Spanic: The virtual experiment was a definite success, sessions were accessible both live and on demand and will be available for the next 3 months for registered attendees and then will be made publicly available. Eminent scientists, not all of whom might have been able to come to Barcelona for the Conference, participated in the event, so it was a great opportunity to hear many of them speaking at the same meeting.

As attendees, the advantage of being able to listen to lectures, debates, and panel discussions once the conference is over is quite relevant. Not only do you not risk missing overlapping sessions, you can tune in and concentrate on more complicated technical lectures at your convenience. As a patient advocate, this is great added value as it allows you to better concentrate on learning and truly understanding the latest research developments. For advocates the educational value is enormous as the lectures can be reviewed and studied later. The virtual exhibition was also useful and well done.

On the downside, an in-presence conference allows networking, in-person meetings, as well as corridor and break time socialization, which was not possible in this 2020 edition of the event. Meeting with other advocates cannot be replaced by virtual contact, and this is a huge part of the EBCC experience which was missed this time. 
Wuerstlein: Prof. Rutgers, could you please summarize news with regard to local therapies?

Rutgers: The EBCC always provides me with a lot of joy. And yes, also this year, in the virtual environment. Professional friends together with the same drive and enthusiasm to thrive for the best for the patients we care of. All together and really multidisciplinary. Positive reinforcements, helpful analysis and criticism, not for the sake of me and myself, but for the patient. For example, the debate on radiotherapy after mastectomy in patients who have positive nodes. It was a sharp debate, lots of arguments, great presentations, and a winner: at the start the pros and cons were equal, after the debate selective radiotherapy on the basis of cancer biology was the winner by 80 versus $20 \%$. And it was all friendly, all "camaraderie" according to Mike Dixon. Indeed, limiting treatment toxicity of local and regional treatments was central in this EBCC. When is surgery in the elderly useful, will it add to the life expectancy, or when is it better to stand back? Prof. Lynda Wyld had an excellent presentation on the "Bridging the Gap" program in the UK on surgery in the elderly. They developed a very nice tool to use in clinical practice to help your decision with your elderly frail patient on whether or not to have surgery. And what about the positive axillary lymph nodes after chemotherapy? Do a complete axillary dissection? Can you target your surgery on the basis of the response in the positive node(s)? The first signals are positive. Yes you can. Prof. Marie Jeanne Vrancken Peeters presented the trial from her institute where they omitted axillary clearance in more the $80 \%$ of patients with initially positive node according to the result of the marked node. After a 3-year follow-up axillary recurrences were very low: short follow-up but a way to manage the axilla worthwhile to study further. In case of a complete remission after upfront chemotherapy on imaging (MRI), can we omit surgery? Since we really want to know if there is a pathological complete response (pCR): if not further chemotherapy is a good and proven option and if a pCR your patient has a favorable prognosis. Well, I can tell you, multiple biopsies from the primary tumor site after chemotherapy will not tell you the whole story. You may miss $20-40 \%$ of residual invasive cancer. So, an excisional biopsy from the - marked - primary tumor bed is still the gold standard (presented by van Duijnhoven et al.). Step by step we will integrate the effect of up-front chemotherapy on the primary cancer and lymph nodes in local and regional treatments. And fortunately, lots of research is ongoing.

\section{Wuerstlein: Prof. Harbeck, could you please summarize news with the aspect of systemic treatments?}

Harbeck: There were so many interesting abstracts submitted that it is very hard to select a few for a more detailed discussion. Let me therefore focus on the plenary session with the selected oral presentations and the press conference before the start of the EBCC. In the plenary session, there were two interesting talks on lobular breast cancer, one by O. Metzger from the MINDACT investigators and one by M. Christgen from the West German Study Group. Even though their specific conclusions regarding the clinical relevance of a high- and a low-risk test result differed, they both demonstrated that we should be aware of the histological type when we interpret these gene expression assay results.

Another interesting talk was the 9-year survival analysis from Neo-ALTTO presented by Dr. Nuciforo on behalf of the Breast International Group. It substantiated the impact of a pCR after neoadjuvant therapy even in an experimental treatment setting as $88 \%$ of patients with a pCR were still alive at 9 years compared to $72 \%$ of those with non-pCR. There was a $4 \%$ better overall long-term survival for patients with dual HER2- blockade - most likely the small study size contributed to this only nonsignificant difference. Nevertheless, the data clearly demonstrated the importance of long-term follow-up in our trials as the investigators were only now able to show that there are no long-term toxicities associated with the treatments.

\section{Wuerstlein: Dr. Spanic, could you please summarize news from the perspective of patients or patient advocacy?}

Spanic: We apply especially to the manifesto and to the ECIBC sessions which represent key areas for advocacy work in our countries and at the EU level.

It was reported that the ECIBC, which has been producing guidelines and a quality assurance scheme for breast cancer services across the continuum of care, is in its final stages. The guidelines are available on the web hub (https:// healthcare-quality.jrc.ec.europa.eu) and are being implemented in some European countries and beyond.

In the manifesto session it was pointed out that health systems vary greatly across Europe, and sometimes even within countries, and that breast care services are not reimbursed or provided in the same way in all regions. This presents a huge challenge to mapping the situation in our countries today and then for Europa Donna advocates to work on these areas in their national advocacy programs while Europa Donna Coalition advocates at the EU level for better services and reimbursement systems. 


\section{Wuerstlein: New research with regard to COVID-19 and breast cancer is/was presented. Could you please comment on this?}

Harbeck, Cortes: In the live COVID-19 session we saw how fast and how well we are able to adapt to such external challenges and collaborate in Europe and beyond for the benefit of our breast cancer patients. I had the opportunity to present an algorithm that we had developed earlier this year with our UK and US colleagues for luminal early breast cancer. If surgical capacities are limited, one way to postpone surgeries in luminal early breast cancer is to put patients on preoperative endocrine therapy. Yet some patients will not benefit from this and need to be taken to surgery right away while others can be maintained for quite some time on their endocrine therapy. The algorithm helps clinicians to make the right decision for their individual patient.

Another important point is the use of e-health, which can support communication between patients and their treatment team, which is particularly important in times of a pandemic when face-to-face contact needs to be limited. We use the interactive CANKADO system (www.cankado.com), which also has a COVID-19 feature.

At the end of the session, Dr. Alessandra Gennari presented first results from a pan-European collaboration which demonstrated in a large cohort of 890 cancer patients with COVID-19 infection that administration of chemotherapy, targeted therapy, or immunotherapy did not worsen mortality. Thus, the consensus at the end of the session was that we should not withhold any diagnostic measure or treatment from our breast cancer patients during this pandemic as delayed diagnosis or inadequate therapy will impact our patients' prognosis even more that the current pandemic.

Spanic: This topic, which was covered in different sessions, is extremely important in terms of what will happen next in terms of screening and perhaps late diagnosis. All experts and health practitioners agreed that suspension of screening programs during spring 2020 across Europe was a necessary precaution which, however, led to a number of negative effects: the first and most immediately perceived was the fall in early breast cancer detection and diagnoses. The use of neoadjuvant therapy while postponing surgery as well as prioritization methods/guidelines for treatment and surgery were also discussed, as was the adequacy of e-medicine and e-health in general in follow-up. Both national and international studies concentrating on the impact of COVID-19 on breast cancer were presented. Once again, it was interesting to notice how each European country had different experiences but similar outcomes.
The feeling is that the outcomes and results can only represent a snapshot of how an emergency was handled and that we will need time to have a clear picture of the true impact the pandemic had on breast cancer in 2020 and will have in the future.

\section{Wuerstlein: What are the final conclusions of the chairs?}

Harbeck: The EBCC is unique with its multidisciplinary approach and its interaction at the conference that encourages this way of approaching breast cancer. The conference thus mirrors the way we treat breast cancer in our breast centers, and it helps to find solutions for difficult-to-treat scenarios such as brain metastases or end of life care. The EBCC thus shows us that optimal care for breast cancer patients involves optimal local or systemic therapy, but also an effective support network for our patients.

As the chair of the EBCC12 I am proud to say that over the October 2-3 weekend alone we had over 6,000 views from all over the world for our 454 recorded and live presentations. This - in my eyes - demonstrates very well that the EBCC continues to connect and educate the global breast cancer community! The virtual conference will stay open for another 3 months. So if you have any colleagues who could not attend the conference, they can still register until the end of 2020 and access all on-demand sessions.

Spanic: One result of this EBCC12 which is important to highlight from a patient advocate perspective is the vote outcome in the debate on mammography screening, which went against the current $\mathrm{EU}$ guidelines for screening of women aged 50-69 years. The Europa Donna head office feels that this could be quite misleading. It would be interesting to know who was present and voting at this session. Under normal circumstances, we would be able to see the attendees in the room and we could better understand the voting dynamic. This was not possible in the virtual setting and it could mean that the vote was skewed by people who might have a special interest. In view of the ECIBC guidelines on screening, this could have quite a negative impact. It could be important to enquire and gather more details on this vote before it gets publicized. Europa Donna will need to be in a position to comment, since we currently very much support population-based mammography screening from 50 to 69 and even from 45 to 74 years. We are also engaged in risk-based screening research as we believe it may have potential in the future, but it certainly cannot and should not replace the current age-based screening programs. Furthermore, our understanding is that women with BRCA or strong 
family history have access to genetic counselling and testing in most countries. We certainly are in favor of that, but we do not consider giving up the current agebased programs to screen only special populations. We need to do both until more research clearly indicates that a change is needed.

Last but not least we sincerely hope that we will all meet in Barcelona in March 2022 for the EBCC13!

\section{Conflict of Interest Statement}

R. Wuerstlein: Agendia, Amgen, Aristo, AstraZeneca, Boehringer Ingelheim, Carl Zeiss, Celgene, Clinsol, Daiichi Sankyo, Eisai, Genomic Health, GlaxoSmithKline, Hexal, Lilly, Medstrom Medical, MSD, Mundipharma, Nanostring, Novartis, Odonate, Onkowissen, Paxman, Palleos, Pfizer, Pierre Fabre, Puma Biotechnology, Riemser, Roche, Sandoz/Hexal, Seattle Genetics, Tesaro Bio, Teva. N. Harbeck reports minority ownership of the Westdeutsche Studiengruppe as well as honoraria for consulting and/ or lectures from Agendia, Amgen, AstraZeneca, BMS, Celgene, Daiichi Sankyo, Genomic Health, Lilly, MSD, Novartis, Odonate, Pierre Fabre, Pfizer, Roche, Samsung, Sandoz/Hexal, and Seattle Genetics (all outside the submitted work). T. Spanic and E.J.T. Rutgers have no conflict of interest to report. J. Cortes: consulting/ advisor: Roche, Celgene, Cellestia, AstraZeneca, Biothera Pharmaceutical, Merus, Seattle Genetics, Daiichi Sankyo, Erytech, Athenex, Polyphor, Lilly, Servier, Merck Sharp\&Dohme, GSK, Leuko, Bioasis, Clovis Oncology, Boehringer Ingelheim, Kyowa Kirin; honoraria: Roche, Novartis, Celgene, Eisai, Pfizer, Samsung Bioepis, Lilly, Merck Sharp\&Dohme, Daiichi Sankyo; institutional research funding: Roche, ARIAD Pharmaceuticals, AstraZeneca, Baxalta GMBH/Servier Affaires, Bayer Healthcare, Eisai, Hoffman-La Roche, Guardant Health, Merck Sharp\&Dohme, Pfizer,
Piqur Therapeutics, Puma C, Queen Mary University of London; stock, patents, and intellectual property: MedSIR; travel, accommodation, expenses: Roche, Novartis, Eisai, Pfizer, Daiichi Sankyo.

\section{Participants}

PD Dr. med Rachel Wuerstlein

Breast Center and CCC Munich

LMU University Hospital Munich

Marchioninistr. 15, DE-81377 Munich (Germany)

rachel.wuerstlein@med.uni-muenchen.de

Prof. Dr. Nadia Harbeck

Breast Center and CCC Munich

LMU University Hospital Munich

Marchioninistr. 15, DE-81377 Munich (Germany)

nadia.harbeck@med.uni-muenchen.de

Dr. Tanja Spanic

Europa Donna Slovenia

Vrazov trg 1, SI-1000 Ljubljana (Slovenia)

tanja.spanic@europadonna.si

Prof. Dr. E.J.T. Rutgers, $M D, P h D, F R C S$

Department of Surgery

Netherlands Cancer Institute

Plesmanlaan 121, NL-1066 CX Amsterdam (The Netherlands) e.rutgers@nki.nl

Dr. Javier Cortes

Head, Breast Cancer Program

IOB Institute of Oncology, Madrid and Barcelona

C/Vilana, 12 planta-1, ES-08022 Barcelona (Spain)

jacortes@vhio.net 